Xiaoping Xu, Shuqian Cai, Wei Chen, Huabin Wang and Junqi Wu*

\title{
Analysis of blood index characteristics in COVID-19 patients and their associations with different outcomes
}

https://doi.org/10.1515/labmed-2020-0090

Received September 18, 2020; accepted February 17, 2021; published online March 9, 2021

\section{Abstract}

Objectives: The clinical implications of different blood indices in patients with coronavirus disease-2019 (COVID-19) were analyzed at different stages.

Methods: We compared blood test results of 17 COVID-19 patients treated in Jinhua Central Hospital between January 1 and March 5, 2020 at different stages. We also compared the initial blood results of 17 COVID-19 patients with 115 influenza virus A/B (Flu A/B)-positive patients, 19 Mycoplasma pneumonia (MP)-positive patients and 50 healthy subjects (HSs).

Results: (1) The white blood cell count (WBC) and absolute neutrophil count (NEU\#) were lower in the SARS-CoV-2 group than in the MP and Flu A/B groups; the eosinophil percentage (EO\%) and absolute eosinophil count (EO\#) were lower in the severe acute respiratory syndromecoronavirus-2 (SARS-CoV-2) group than in the MP group $(\mathrm{p}<0.05)$. (2) Aspartate aminotransferase (AST) levels were significantly lower when patients were discharged from the hospital $(\mathrm{p}<0.05), \mathrm{EO} \%$ and EO\# recovered at discharge, and returned to normal levels during follow-up ( $\mathrm{p}<0.05)$. (3) When the throat swab was nucleic acid-negative but the stool was still positive, lymphocyte percentage (LY\%) and absolute lymphocyte count (LY\#) decreased ( $\mathrm{p}<0.05)$. (4) As the cycle threshold (Ct) value of the nucleic acid increased or decreased, EO\# showed a consistent trend.

*Corresponding author: Junqi Wu, Department of Biochemistry, Affiliated Jinhua Hospital, Zhejiang University School of Medicine, Jinhua, Zhejiang Province, 321000, P.R. China; and Department of Biochemistry, Clinical Laboratory, Affiliated Jinhua Hospital, Zhejiang University School of Medicine, 365 Renmin East Road, Jinhua City, Zhejiang Province, P.R. China, Phone: +86 18757608330, Fax: +86 0579 82552088, E-mail: jhwujunqi@sina.com

Xiaoping Xu, Shuqian Cai, Wei Chen and Huabin Wang, Department of Clinical Laboratory, Affiliated Jinhua Hospital, Zhejiang University School of Medicine, Jinhua, Zhejiang Province, P.R. China. https:// orcid.org/0000-0002-9090-7380 (H. Wang)
Conclusions: Blood cell count indices upon hospital admission could be helpful to give some tips of diagnosis of SARS-CoV-2-infection, Flu A/B-infection and MP-infection; AST and EO\# could be used to predict the outcome of patients. Feces turned negative for nucleic acid more slowly than throat swabs; LY\# was lower during the fecalpositive period and low $\mathrm{Ct}$ values of fecal nucleic acid were negatively associated with the patient's recovery level.

Keywords: COVID-19; eosinophil; nucleic acid; SARS-CoV-2.

\section{Introduction}

In December 2019, a coronavirus disease-2019 (COVID-19) epidemic broke out in Wuhan, which quickly spread worldwide [1]. As of March 11, 2020, a total of 118,000 confirmed patients worldwide have been reported, in more than 110 countries, causing more than 4,300 deaths. The WHO has announced that the COVID-19 epidemic constitutes a global pandemic. Severe acute respiratory syndrome-coronavirus-2 (SARS-CoV-2) is a single-stranded positive-strand RNA virus and is the seventh member of the coronavirus family. It has $79.5 \%$ homology with SARS-CoV and has $96 \%$ homology with the bat coronavirus in the bat Yunnan chrysanthemum. [2].

SARS-CoV-2 can be detected in nasal swabs, throat swabs, sputum specimens, alveolar lavage fluid, serum/ plasma and fecal of infected persons by nucleic acid amplification [3, 4]. However, because most patients suffer from dry cough, it is difficult to obtain sputum. Alveolar lavage fluid sampling is likely to cause aerosol transmission, and early concentrations of viral nucleic acids in the blood are too low. Therefore, throat swabs and fecal specimens are more commonly sampled for diagnosing and monitoring patients. According to the latest treatment guidelines of the Ministry of Health for COVID-19, pharyngeal swab nucleic acid tests must be negative for two consecutive times ( $24 \mathrm{~h}$ interval) to confirm patient recovery. However, some patients had nucleic acidpositive feces that lasted for a long time. During this period, the patient's condition basically recovered. Fecal samples should be tested to exclude a potential alternative 
route of transmission that is unknown at this stage [5]. It is controversial whether patients with negative throat swabs but positive feces could be discharged. It is very meaningful to analyze whether changes in blood indices during the fecal-positive period are associated with patient outcome.

SARS-CoV-2 nucleic acid testing is the golden standard for the diagnosis of COVID-19 patients, but the virus load is low in the early stage of the disease, the nasopharynx is not the focal point of early infection and the operation is complicated, so throat swab tests have high clinical false negative rates. Antiviral antibody detection can also assist diagnosis; Xi Mo et al. [6] believed that it takes a period of time from SARS-CoV-2 infection to antibody production. Serum antibody detection of immunoglobulin G/M (IgG/M) lags behind nucleic acid detection. Positive antigen tests can also provide strong pathogenic evidence, but current methods for antigen testing are less mature. In the early stage of COVID-19, fever and dry cough are the primary manifestations. Winter is the season of high influenza virus incidence, and influenza virus A/B (Flu A/B)-infected patients also develop fever and dry cough. Patients with Mycoplasma pneumoniae (MP) infection also have flu-like symptoms in the early stage. Early rapid diagnosis of COVID-19 patients will make up for missed detections due to false negatives in nucleic acid tests and help clinicians to gain time for patient treatment. Similarly, with the development of the patient's condition, if some blood indices are significantly associated with patient outcome, they will be helpful to evaluate the patient's condition.

We conducted a statistical analysis of the blood cell count, biochemical test results, and C-reactive protein (CRP) levels of newly diagnosed COVID-19 patients, MP-positive patients, Flu A/B-positive patients, and healthy people, and found that some of the blood indices show significant changes between groups. Moreover, we analyzed the differences in blood cell count and biochemical indices between each patient's initial diagnosis, discharge, and follow-up visit. We found that blood indices, except aspartate aminotransferase (AST) levels, did not return to normal values when the patient was discharged, and most of the indices had returned to normal levels at follow-up after 14 days. The observed trends were consistent; we found that some blood indices of fecal-positive patients with positive or negative throat swabs were different. For example, absolute lymphocyte count (LY\#) decreased significantly during the fecalpositive period.

\section{Materials and methods}

\section{Sample collection}

Between January 25, 2020 and March 5, 2020, 17 patients (10 males and seven females) were diagnosed with SARS-CoV-2 by throat swab nucleic acid testing at the Jinhua City Center for Disease Control and Prevention. Their median age was 37 years (interquartile range, 29-50 years). Except for a 67-year-old woman with cholecystitis, no patients had concomitant diseases and no complications occurred during hospitalization. We also collected data of 19 MP IgM-positive patients, $115 \mathrm{Flu}$ A/B-positive patients, and 50 healthy controls (HSs) treated during the same period. There was no cross-infection between the groups, and there were no statistical differences in age distribution, gender ratio, and concomitant disease between the latter three groups and the COVID-19 patient group.

\section{Laboratory testing}

All tested blood indices were WHO-recommended test items [7]. Blood, oral swab, and stool sampling and testing were performed in accordance with the standard operating procedures of the laboratory. Biochemical items (total protein [TP], albumin [ALB], total bilirubin [TBIL], aspartate aminotransferase [AST], alanine aminotransferase [ALT], lactate dehydrogenase [LDH], creatinine [CREA], blood urea nitrogen [BUN], and amylase [AMY]) were tested on an AU5821 reader (Beckman, Japan); blood cell count items (white blood cell count [WBC], absolute neutrophil count [NEU\#], absolute lymphocyte count [LY\#], absolute monocyte count [MN\#], absolute eosinophil count [EO\#], absolute basophil count [BA\#], neutrophil percentage [NEU\%], lymphocyte percentage [LY\%], monocyte percentage [MN\%], eosinophil percentage $[\mathrm{EO} \%]$, and basophil percentage $[\mathrm{BA} \%]$ ) were measured on an XE-2100 reader (SYSMEX, Japan); Flu A, Flu B, and SARS-CoV-2 nucleic acid detection was performed with virus nucleic acid amplification fluorescence qualitative kits (Zhijiang, China) on an AB7500 Real time PCR (Polymerase chain reaction) system (Life Technology, USA). All results were collected through the laboratory information system, and patient epidemiological data were collected through electronic medical records.

\section{Statistical methods}

Statistical analysis was performed using SPSS 26.0 software. Normality was tested using the independent-sample KolmogorovSmirnov test. Normally distributed data are represented as average \pm standard deviation. Comparisons between multiple groups were made by analysis of variance; pairwise comparisons were performed by $t$ test. Non-normally distributed data are expressed as median (Q25-Q75). Comparisons between groups were performed using the Kruskal-Wallis test and pairwise comparisons were performed using the Mann-Whitney U test. Differences are considered to be statistically significant at $\mathrm{p}<0.05$. 


\section{Results}

\section{General condition and outcome of the patient}

The median age of the 17 COVID-19 patients was 37 years (interquartile range, 29-50 years). Ten males and seven females were included. All patients were affected by SARS-CoV-2 because they had been to Wuhan or had been in contact with relatives and/or friends who had been to Wuhan. The median incubation period before symptom onset was six days (interquartile range, 3.5-9 days) according to the patients' medical histories.

First symptom: in 11/17 patients, the first symptom was fever without a source; four of them subsequently developed dry cough, two had muscle aches and weakness, one had palpitations, and one had both dry cough and loose stool; in $3 / 17$ patients, the first symptom was pharynx pain, and a sore throat/fever appeared 2-5 days later; in 2/17 patients, the first symptoms were cough and fever; in 1/17 patient, the first symptom was cough without fever. 14/17 had pulmonary inflammation. 10/17 had ground glass opacity, 2/17 had unilateral shadows, 2/17 had bilateral shadows. The (computerized tomography) CT results show that 14/17 had pulmonary inflammation. 10/17 had ground glass opacity, 2/17 had unilateral shadows, 2/17 had bilateral shadows.

Upon hospital admission of all patients, throat swab SARS-CoV-2 nucleic acid test results were positive; the median time for the throat swab to turn negative was eight days (interquartile range, 2.75-13.5 days); the fecal nucleic acid test was not carried out at the beginning. The duration of fecal positivity is unknown. In 10/17 patients, the stool and throat swabs remained SARS-CoV-2 nucleic acidnegative during hospitalization, and fecal positivity appeared in $7 / 17$ patients during hospitalization. The patients' temperature continued to be normal when fecal specimens were nucleic acid-positive, and lung CT showed that in the stage of inflammation subsiding, the patients' physical signs were stable. Among these seven cases, throat swabs of six cases turned nucleic acid-negative before the feces did, and in the remaining case feces turned nucleic acid-negative before throat swabs did. Twelve people were discharged from the hospital for two weeks and returned for follow-up. One person was found fecalpositive (fecal nucleic acid was not detected before hospital discharge). The patient remained isolated for two weeks at home, and during follow-up feces was nucleic acid-negative. The throat swabs and feces of the remaining 11 patients were negative for nucleic acid. All 17 COVID-19 patients were discharged (in three patients, the feces was still positive upon hospital discharge). The median length of hospital stay was 11 days (interquartile range, 6-21 days), and anti-SARS-CoV-2 antibody IgM and IgG levels were measured during follow-up in 12 patients two weeks after discharge (Figure 1).

Upon hospital admission, the throat swab nucleic acid test was positive in all 17 patients; the red vertical line indicates when the fecal nucleic acid test came in use.
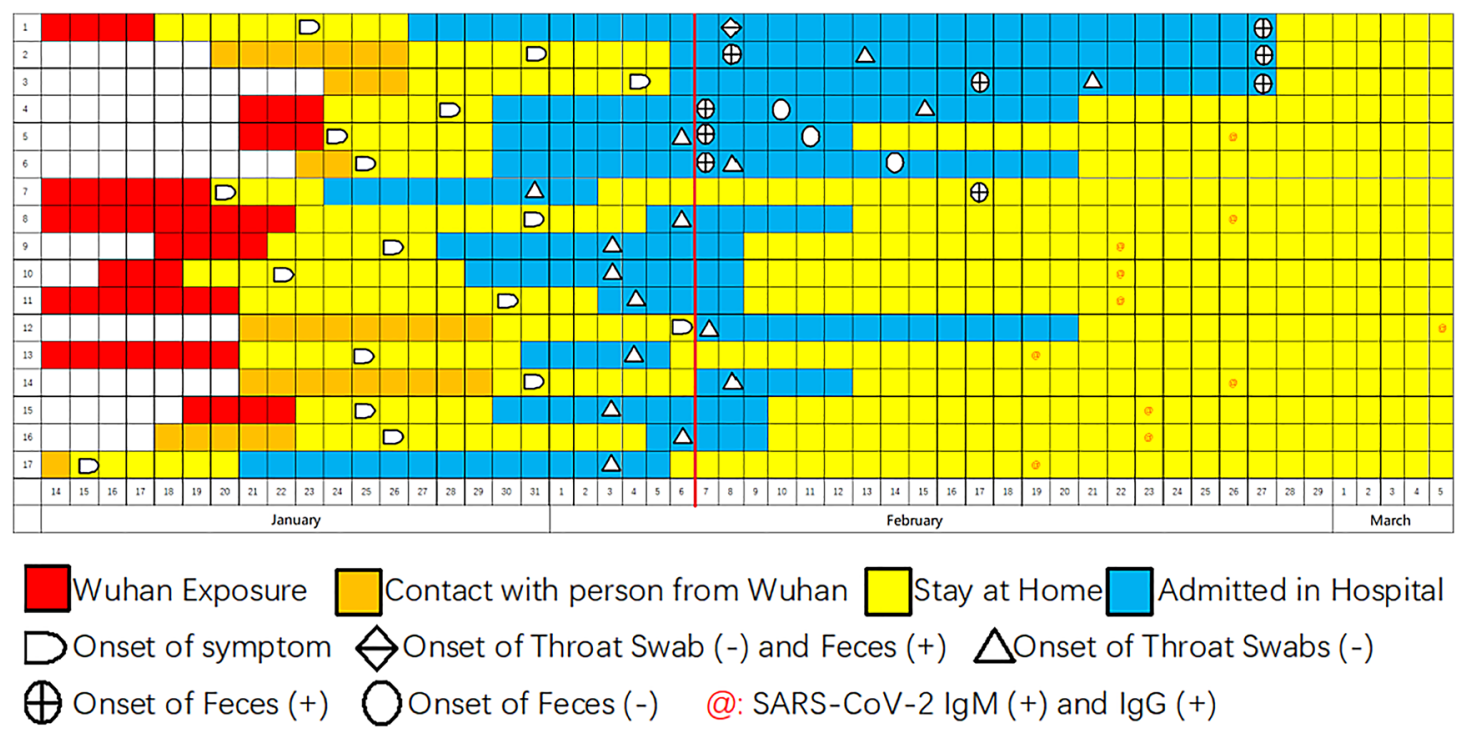

Figure 1: Summary of COVID-19 patient incubation periods and times of discharge and admission. 


\section{WBC, NEU\#, NEU\%, LY\%, EO\%, and EO\# were different from those of MP-positive patients and flu A/B-positive patients at the initial diagnosis}

We compared the blood cell counts, biochemical tests, and CRP levels upon hospital admission between the HS group, the MP-positive group, the Flu A/B-positive group, and the SARS-CoV-2-positive group (Table 1). Compared with HSs, the changes in each group are basically consistent with the guidelines for the treatment of related viruses or bacteria $[8,9]$. (1) WBC: The mean WBC of patients in the SARS-CoV2 group was lower than in the HS group ( $p>0.05$ ), $25 \%$ of the values were low; the mean of the Flu A/B group was higher than that of the HS group ( $>0.05$ ), $22 \%$ of the values were high; the mean value of the MP group was higher than that of the HS group ( $p<0.05), 15 \%$ of the values were high. The WBC was lower in the SARS-CoV-2 group than in the MP and Flu A/B groups ( $\mathrm{p}<0.05)$. (2) NEU\%: The mean NEU\% of patients in the SARS-CoV-2 group was higher than in the HS group ( $p>0.05), 24 \%$ of the values were higher; the average of the Flu A/B group was higher than that of the HS group ( $<<0.05$ ), $52 \%$ of the values were high; and the mean value of the MP group was higher than that of the HS group ( $p>0.05$ ), $40 \%$ of the values were high. NEU\% was lower in the SARS-CoV-2 group than in the Flu A/B group $(\mathrm{p}<0.05)$. (3) LY\%: The mean LY\% of patients in the SARS-CoV-2 group was lower than in the HS group (p>0.05), 18\% of the values were low; the mean in the Flu A/B group was higher than that in the HS group ( $\mathrm{p}<0.05$ ), $52 \%$ of the values were biased; and the average in the MP group was higher than that in the HS group $(\mathrm{p}<0.05)$, which was higher than $35 \%$. $\mathrm{LY} \%$ in the SARS-CoV-2 group was higher than in the Flu A/B group (p<0.05). (4) EO\%: The mean EO\% of patients in the SARS-CoV-2 group was lower than in the HS group $(p<0.05), 71 \%$ of the values were low; the average of the Flu A/B group was lower than that of the HS group $(p<0.05), 52 \%$ of the values were low; and the mean of the MP group was lower than that of the HS group $(p<0.05), 30 \%$ of the values were low. EO $\%$ was lower in the SARS-CoV-2 and Flu A/B groups than in the MP group $(\mathrm{p}<0.05)$. (5) NEU\#: The mean NEU\# was the lowest in the SARS-CoV-2 group (SARS-CoV-2 vs. HS, $\mathrm{p}<0.05), 24 \%$ of the values were low; it was higher in the Flu A/B group than in the HS group ( $p>0.05$ ), 39\% of the values were high; and the mean of the MP group was higher than that of the HS group ( $p<0.05), 30 \%$ of the values were high. NEU\# in the SARS-CoV-2 group was lower than in the MP and Flu A/B groups ( $\mathrm{p}<0.05)$. (6) EO\#: The mean of EO\# patients in the SARS-CoV-2 group was lower than in the HS group $(p<0.05)$; the mean of the Flu A/B group was lower than that of the HS group $(p<0.05)$; and the mean of the MP group was lower than that of the HS group ( $p>0.05)$. EO\# in the SARS-CoV-2 group was lower than in the MP and Flu A/B groups $(\mathrm{p}<0.05)$. (7) CRP: The mean of CRP patients in the SARS-CoV-2 group was higher than in the HS group $(p<0.05)$; the mean of the Flu A/B group was higher than that of the HS group ( $>>0.05)$; and the mean of the MP group was higher than that of the HS group ( $p>0.05)$. CRP in the SARS-CoV-2 group was higher than in the MP and Flu A/B groups $(\mathrm{p}<0.05)$.

\section{Comparison of blood indices of 17 COVID-19 patients upon admission, discharge, and follow-up (13 patients were fecal-negative and 11 patients were followed up at two weeks)}

Based on the results in Table 1, the indices that are significantly different between the HS group and other groups were selected, and these were compared with the values upon admission. EO\% and EO\# were higher at discharge $(p<0.05)$, but did not reach normal values $(\mathrm{p}<0.05)$; two weeks after discharge, they were basically recovered to normal levels. MN\% was still high upon discharge ( $p>0.05)$, but recovered to normal levels within two weeks; LY\# was still low upon discharge ( $p>0.05)$, but returned to normal levels within two weeks. ALT levels were still high upon discharge $(\mathrm{p}>0.05)$, and did not decrease significantly within two weeks ( $>0.05)$; AST levels were significantly lower upon discharge $(\mathrm{p}<0.05)$ and did not change significantly within two weeks ( $>0.05$ ). There was no significant difference with normal values ( $p>0.05)$. LDH levels were still high upon discharge ( $>0.05$ ), and remained high two weeks after discharge (Figure 2).

\section{Number of eosinophils and $\mathrm{Ct}$ value of nucleic acid}

Data were collected of patients for whom more than two simultaneous measurements of the $\mathrm{Ct}$ value and EO\# were performed. We found that as the Ct value increases, EO\# also gradually increases; as the $\mathrm{Ct}$ value decreases, EO\# also gradually decreases. These observed trends were relatively consistent. 
Table 1: Comparison of blood indices upon admission between HS, MP-positive patients, Flu A/B-positive patients and SARS-CoV-2-positive patients.

\begin{tabular}{|c|c|c|c|c|}
\hline Groups & A & B & C & D \\
\hline WBC & $5.53 \pm 1.31$ & $7.19 \pm 2.60^{\mathrm{a}}(15 \%)$ & $6.37 \pm 2.43(22 \%)$ & $4.76 \pm 1.74^{b, c}(25 \%)$ \\
\hline NEU\% & $58.38 \pm 7.48$ & $65.19 \pm 13.91(40 \%)$ & $71.90 \pm 14.75^{\mathrm{a}}(52 \%)$ & $62.04 \pm 11.93^{c}(24 \%)$ \\
\hline $\mathrm{LY} \%$ & $33.77 \pm 7.40$ & $25.06 \pm 11.91^{\mathrm{a}}(35 \%)$ & $19.00 \pm 12.12^{\mathrm{a}}(52 \%)$ & $28.98 \pm 9.91^{\mathrm{C}}(18 \%)$ \\
\hline MN\% & $5.27 \pm 1.28$ & $7.83 \pm 2.88^{\mathrm{a}}(30 \%)$ & $8.32 \pm 3.16^{\mathrm{a}}(26 \%)$ & $8.32 \pm 2.84^{\mathrm{a}}(29 \%)$ \\
\hline EO\% & $2.05(1.5-2.83)$ & $1.25(0.3-2.63)^{\mathrm{a}}(30 \%)$ & $0.3(0-0.7)^{\mathrm{a}, \mathrm{b}}(52 \%)$ & $0.2(0-1.05)^{\mathrm{a}, \mathrm{b}}(71 \%)$ \\
\hline $\mathrm{BA} \%$ & $0.2(0.13-0.38)$ & $0.2(0.05-0.4)$ & $0.2(0.2-0.4)$ & $0.2(0-0.3)$ \\
\hline NEU\# & $3.25 \pm 1.00$ & $4.87 \pm 2.18^{\mathrm{a}}(30 \%)$ & $4.79 \pm 2.43(39 \%)$ & $2.93 \pm 1.16^{b, c}(24 \%)$ \\
\hline LY\# & $1.82 \pm 0.43$ & $1.63 \pm 0.60(70 \%)$ & $1.04 \pm 0.54^{\mathrm{a}, \mathrm{b}}(61 \%)$ & $1.40 \pm 0.77^{\mathrm{a}}(41 \%)$ \\
\hline MN\# & $0.29 \pm 0.92$ & $0.55 \pm 0.28^{a}$ & $0.50 \pm 0.22^{a}$ & $0.40 \pm 0.19$ \\
\hline EO\# & $0.11(0.07-0.16)$ & $0.07(0.02-0.19)$ & $0.02(0-0.03)^{a, b}$ & $0.01(0-0.06)^{a, b}$ \\
\hline BA\# & $0.01(0.01-0.02)$ & $0.2(0.1-0.4)$ & $0.01(0.01-0.02)$ & $0.01(0-0.02)$ \\
\hline CRP & $3.2(1.1-4.35)$ & $3.85(0.5-11)(25 \%)$ & $3.7(0.5-34)(43 \%)$ & $8.6(1.7-12)^{a, b, c}(53 \%)$ \\
\hline UREA & $5.01 \pm 0.78$ & $4.28 \pm 0.93(35 \%)$ & $5.11 \pm 2.52(30 \%)$ & $4.66 \pm 0.73(23 \%)$ \\
\hline CREA & $68.31 \pm 15.3$ & $64.04 \pm 13.99(32 \%)$ & $69.93 \pm 42.00(40 \%)$ & $67.99 \pm 13.33(24 \%)$ \\
\hline ALT & $21.27 \pm 9.61$ & $27.74 \pm 19.91(14 \%)$ & $37.40 \pm 22.32^{\mathrm{a}}(19 \%)$ & $34.40 \pm 18.18^{\mathrm{a}}(29 \%)$ \\
\hline AST & $22.79 \pm 4.95$ & $32.12 \pm 8.21^{\mathrm{a}}(15 \%)$ & $40.00 \pm 15.48^{\mathrm{a}}(13 \%)$ & $35.41 \pm 11.61^{\mathrm{a}}(6 \%)$ \\
\hline $\mathrm{LDH}$ & $169.05 \pm 27.85$ & $354.87 \pm 119.34^{\mathrm{a}}(33 \%)$ & $428.09 \pm 97.63^{\mathrm{a}}(20 \%)$ & $482.18 \pm 127.66^{\mathrm{a}}(29 \%)$ \\
\hline AMY & $60.35 \pm 16.91$ & $69.17 \pm 11.36$ & $79.70 \pm 18.04^{a}$ & $71.06 \pm 15.67$ \\
\hline TBIL & $13(0.48-16.98)$ & $15.7(13.33-22.68)(20 \%)$ & $9.9(8.4-13.3)^{b}$ & $12.2(10.7-17.1)(18 \%)$ \\
\hline TP & $72.27 \pm 3.13$ & $69.24 \pm 6.44$ & $77.11 \pm 5.27^{\mathrm{a}, \mathrm{b}}$ & $74.04 \pm 5.62$ \\
\hline ALB & $45.06 \pm 2.25$ & $47.41 \pm 5.46$ & $42.95 \pm 3.73$ & $43.42 \pm 4.04$ \\
\hline
\end{tabular}

A: HS group; B: MP-positive group; C: Flu A/B-positive group; D: SARS-CoV-2-positive group. Parentheses indicate the proportion of outliers not in the reference range. ${ }^{a} p<0.05$ compared with the control group; ${ }^{b} p<0.05$ compared with the MP group; ${ }^{c} p<0.05$ compared with the Flu A/B group.

\section{Partial blood routine and biochemical tests were performed during the throat swab- positive (TS+) and throat swab-negative but fecal-positive (TS-F+) periods}

Different stages of infection were compared (Table 1), and the following interesting results were obtained. (1) The mean WBC of the TS-F+ group was higher than that of the TS+ group $(\mathrm{p}<0.05)$. (2) The mean NEU\# of the $\mathrm{TS}-\mathrm{F}+$ group was higher than that of the TS+ group $(p<0.05)$. (3) The mean NEU\% of the TS-F+ group was higher than that of the TS+ group $(\mathrm{p}<0.05)$. (4) The mean LY\% of the TS-F+ group was lower than that of the TS+ group ( $\mathrm{p}<0.05)$. (5) The mean LY\# in the TS-F+ group was lower than that in the TS+ group $(\mathrm{p}<0.05)$. (6) The mean erythrocyte sedimentation rate (ESR) in the TS-F+ group was higher than that in the TS+ group $(\mathrm{p}<0.05)$. (7) The mean ALT levels in the TS-F+ group were higher than in the TS+ group $(\mathrm{p}<0.05)$. (8) The mean AST levels in the TS-F+ group were higher than in the TS+ group $(\mathrm{p}<0.05)$. In summary, WBC, NEU\%, NEU\#, ESR, ALT, and AST were higher in the TS-F+ than in the TS+ group $(\mathrm{p}<0.05)$. $\mathrm{LY} \%$ and LY\# were lower in the TS-F+ group than in the TS+ group $(\mathrm{p}<0.05)$.

\section{Discussion}

The incubation period and symptoms of 17 COVID-19 patients were in agreement with those reported in related literature [5]. A total of 39 COVID-19 patients in our hospital and surrounding hospitals have been found to be negative for the Flu A/B. Considering the current high incidence of influenza, it is speculated that the probability of overlap of infection with influenza virus and SARS-CoV-2 is low. The symptoms of patients with early SARS-CoV-2 infection are not obvious, and lung CT does not give strong indications of inflammation. The nucleic acid positivity rate of throat swab sampling is low. COVID-19 patients are easily confused with other patients with common pneumonia. Ai et al. [10] considered that it is important to strengthen the differential diagnosis of pneumonia caused by COVID-19 and other pathogen infections. They performed A and B flow, respiratory syncytial viral (RSV), and differential diagnosis of mycoplasma and nasal virus in 53 patients with suspected COVID-19 by RT-PCR and met genomics next generation sequencing (mNGS). It was considered that the detection of other respiratory pathogens during the winter period, when people are prone to pneumonia, could not be used as an exclusion criterion for COVID-19. Some 

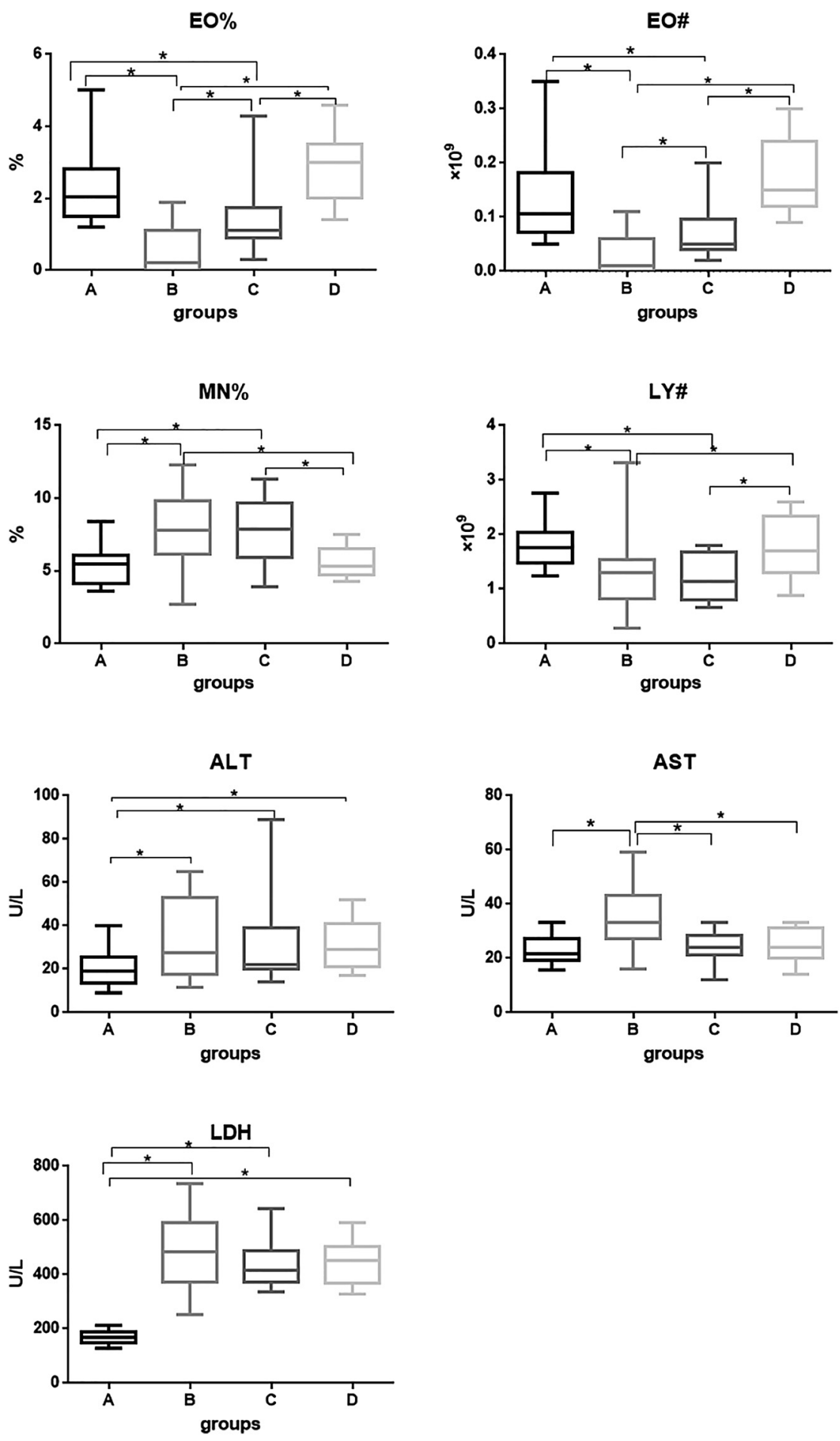

Figure 2: Comparison of the blood indices EO $\%$, EO\#, MN\%, LY\#, ALT, AST, and LDH at the time of admission, discharge, and follow-up and in healthy subjects. A: HS group; B: COVID-19 patients upon admission; C: COVID-19 patients upon discharge; D: COVID-19 patients 14 days after discharge. ${ }^{*} p<0.05$.

blood routine indices can help identify Flu A/B-infection and MP-infection, which is helpful for the initial screening of COVID-19 patients during the outbreak. COVID-19 patients can be initially identified based on WBC, NEU\%, NEU\#, LY\%, and Flu A/B positivity; MP-positive patients can be initially identified based on $\mathrm{WBC}$, NEU\#, $\mathrm{EO} \%$, and EO\# (Table 1). Virus infection will not cause great changes in CRP. However, CRP levels were highest in COVID-19 patients than other groups, one possible explanation for this phenomenon is the overproduction of inflammatory cytokines. Cytokines fight against the pathogen but when the system hyperactivates, it can damage lung tissue. CRP production is induced by cytokines and tissue destruction, but this is only the result of the first-diagnosed patient, and 
there is no big change from the normal reference interval yet [11]. No significant differences in biochemical indices were observed with HSs, and they cannot be used for diagnosis.

AST levels were positively associated with patient outcome, and high ALT levels were associated with poor prognosis (Figure 2). Zhang et al. [12] believe that (angiotensin converting enzyme) ACE2 as a key receptor is not only expressed on tracheal epithelial cells and lung AT2 cells, but also in absorbable intestinal epithelial cells, such as colon and ileum. This finding suggests that the virus may invade the liver through the digestive tract or blood circulation. As ACE2 is highly expressed on bile duct cells, which suggests that SARS-CoV-2 may directly bind to ACE2-positive bile duct cells, resulting in liver dysfunction. Anna et al. [13] find that mildly abnormal plasma Liver Function Tests, especially AST and ALT, are frequently observed in patients with COVID-19 on admission and are associated with severe disease and increased inflammatory markers. LDH levels increased rapidly after symptom onset, and LDH levels were still high after recovery. Since the total lactate dehydrogenase was detected in our hospital, LDH contains five isoenzymes, and the content of LDH3 is the highest in the lungs (lung injury can cause LDH3 levels to increase). The level of LDH in patients with no lung inflammation was still higher than that in normal people, as the AST and ALT levels of 17 patients were increased, suggesting that virus may invade the liver through the digestive tract or blood circulation. It also caused the elevation of LDH4 and LDH5. As the lungs were still recovering from inflammation, the increase in LDH levels is speculated to be related to lung or liver damage, so patients need to return for follow-up evaluation after two weeks. LY\# was still low upon discharge, and it returned to normal values after 14 days, which may be indirectly caused by the viral infection [14]. EO\% and EO\# were significantly lower at the time of disease onset, which may be due to suppression under stress conditions, or it may be the result of endogenous glucocorticoid secretion and the production of chemokines at the site of infection [15]. Degranulation of basophils and monocytes is involved in eosinopenia sequestration at the site of infection [16]. EO\% and EO\# were still lower than normal levels when the patient was discharged, and they returned to normal values within 14 days. Eosinophils were significantly associated with patient outcome, and can hence be used to assess the patient's recovery level. At the same time, EO\# changes with changing $\mathrm{Ct}$ values and they have better consistency, indirectly reflecting that EO\# can be used to roughly assess dynamic changes in SARS-CoV-2 infection (Table 2). Caroline et al. studied the relationship between EO and Ct values, and they found several lines of investigation have indicated a role for eosinophilic leukocytes in limiting virus infectivity and promoting virion clearance [17]. Liu et al. compared the relationship between $\mathrm{EO}$ and $\mathrm{Ct}$ values, and they believed that increasing eosinophils may be an indicator of COVID-19 improvement [18].

In addition to the respiratory tract, viruses often invade the human digestive tract, and certain parenteral viruses can bypass physical barriers to infect susceptible cells in the digestive tract [19]. Like SARS-CoV, SARS-CoV-2 has been confirmed to infect ciliary bronchial epithelial cells and type II lung cells with angiotensin converting enzyme 2 (ACE2) as a receptor, thereby causing lung disease [20-22]. Holshue et al. [23] detected SARS-CoV-2 nucleic acid in the stool of confirmed patients. Nanshan Zhong's team isolated a live fecal virus [24]; they believe that fecal viral nucleic acid positivity and the presence of virus are two different things, and further research is needed to determine whether fecal viral nucleic acid is contagious. During treatment, seven patients with throat swab nucleic acid continued to be positive after feces turned negative for viral nucleic acid (Figure 1). SARS-CoV-2 may infect the digestive tract by some means after colonization of the

Table 2: Ct values of nucleic acid in throat swabs and feces and EO\#.

\begin{tabular}{|c|c|c|c|c|c|c|c|c|c|c|c|c|c|}
\hline \multirow{2}{*}{$\begin{array}{l}\text { Patient } \\
\text { Date }\end{array}$} & \multicolumn{2}{|c|}{ P1 } & \multicolumn{2}{|c|}{ P2 } & \multicolumn{4}{|c|}{ P3 } & \multicolumn{3}{|c|}{ P4 } & \multicolumn{2}{|c|}{ P5 } \\
\hline & Do & D10 & D4 & D5 & D0 & D7 & D12 & D16 & D0 & D7 & D10 & DO & D7 \\
\hline TS-Ct & 27 & 37.7 & 32.4 & 35.9 & 30 & 33.2 & 38.3 & 40.9 & 30 & 36.3 & 37.2 & 28.0 & 38.8 \\
\hline EO\# $\left(\times 10^{9}\right)$ & 0.01 & 0.03 & 0.06 & 0.08 & 0.06 & 0.18 & 0.20 & 0.24 & 0.0 & 0.02 & 0.03 & 0.06 & 0.11 \\
\hline \multirow{2}{*}{$\begin{array}{l}\text { Patient } \\
\text { Date }\end{array}$} & \multicolumn{4}{|c|}{ P2 } & \multicolumn{3}{|c|}{ P3 } & \multicolumn{3}{|c|}{ P4 } & \multicolumn{3}{|c|}{ P6 } \\
\hline & \multicolumn{2}{|c|}{ D6 } & D7 & D12 & & D4 & D5 & & D13 & D19 & & D12 & D15 \\
\hline $\mathrm{FS}-\mathrm{Ct}$ & \multicolumn{2}{|c|}{36.8} & 32.7 & 31.3 & \multicolumn{2}{|r|}{37.6} & 40.1 & \multicolumn{2}{|r|}{33.8} & 31.3 & \multicolumn{2}{|r|}{37.9} & 37.9 \\
\hline EO\# $\left(\times 10^{9}\right)$ & \multicolumn{2}{|c|}{0.08} & 0.06 & 0.05 & \multicolumn{2}{|r|}{0.15} & 0.18 & \multicolumn{2}{|r|}{0.03} & 0.05 & \multicolumn{2}{|r|}{0.04} & 0.04 \\
\hline
\end{tabular}

TS, throat swab; FS, fecal sample. 
respiratory tract, as six patients had pharyngeal infection. The throat swab first turned negative for nucleic acid, and fecal nucleic acid positivity lasted for a longer time. During this period, the throat swab remained negative for nucleic acid, and the digestive tract SARS-CoV-2 live virus did not infect the respiratory tract again.

The effects of SARS-CoV-2 on the human respiratory and digestive tracts are inconsistent. From the analysis of the symptoms, it seems patients gradually recover during the fecal-positive period. The body temperature is normal, and the lung CT shows inflammation subsiding, which seems to be similar to normal people. Moreover, feces continue to be positive for nucleic acid. Comparing blood indices in two periods, multiple differences were observed. LY\# did not change during disease progression, and other indices also showed that they were still at the level of inflammation. Fecal nucleic acid testing was not carried out at the beginning of the epidemic. The first three cases of COVID-19 patients were not tested for fecal nucleic acid when they were discharged. One patient was found to be positive for fecal nucleic acid at the two-week follow-up. Due to the patient's self-isolation after discharge, the fecal-oral transmission could not be further evaluated. The patient's blood indices AST, ALT, and NE\# were higher than before discharge, LY\# decreased, and EO\# was still at the level before discharge, which is consistent with the results in Table 3. The patient tested negative for fecal nucleic acid after seven days. It is still unknown whether fecal-oral transmission is an alternative infection route. Three of the seven fecal-positive patients remained positive

Table 3: Comparison of some blood indices between the throat swab-positive (TS+) group and the throat swab-negative but fecalpositive (TS-F+) group.

\begin{tabular}{lrr}
\hline Item & TS $+(\mathbf{n}=9)$ & TS-F+ $(\mathbf{n}=7)$ \\
\hline WBC & $5.38 \pm 1.16$ & $7.36 \pm 1.28^{\mathrm{a}}$ \\
NEU\% & $56.61 \pm 8.52$ & $71.17 \pm 5.66^{\mathrm{a}}$ \\
LY\% & $32.37 \pm 7.44$ & $20.59 \pm 5.12^{\mathrm{a}}$ \\
MN\% & $8.69 \pm 2.37$ & $7.1 \pm 1.96$ \\
EO\% & $0.94 \pm 1.98$ & $0.7 \pm 0.44$ \\
NEU\# & $3.06 \pm 0.91$ & $5.27 \pm 1.19^{\mathrm{a}}$ \\
LY\# & $1.73 \pm 0.50$ & $1.49 \pm 0.35^{\mathrm{a}}$ \\
MN\# & $0.47 \pm 0.18$ & $0.51 \pm 0.12$ \\
EO\# & $0.06(0.02-0.19)$ & $0.06(0.02-0.08)$ \\
CRP & $3.9(0.5-7.42)$ & $7.5(1.35-27.48)$ \\
UREA & $4.06(3.62-4.87)$ & $3.22(2.88-6.08)$ \\
CREA & $80.56 \pm 12.28$ & $71.03 \pm 9.83$ \\
ALT & $34.49 \pm 15.47$ & $58.43 \pm 20.57^{\mathrm{a}}$ \\
AST & $28.89 \pm 5.99$ & $35.86 \pm 8.43^{\mathrm{a}}$ \\
LDH & $464.44 \pm 97.69$ & $438.57 \pm 25.49$ \\
\hline
\end{tabular}

${ }^{a} \mathrm{p}<0.05$ compared with the TS $+\mathrm{F}-$ group. until discharge from the hospital, and the fetal nucleic acid Ct value was low. Alvaro et al. believed that RT-PCR assays of fecal specimens, is an important step to control infection, suggesting that samples remained positive for SARS-CoV-2 RNA longer time then respiratory tract samples [25]. Chen et al. introduced one case, this case indicates that the virus can proliferate in the digestive tract and potentially undergo fecal-oral transmission [26]. The patients were strongly positive for antiviral IgG and IgM. Persistence of fecal positivity may be due to higher secretion levels of ACE2 in the digestive tract. IgM is produced during common respiratory viral infections, and 12 SARS-CoV-2 patients who were followed up after two weeks SARS-CoV-2 were still positive for IgM, indicating that the duration of IgM presence was longer than the actual infection.

The SARS-CoV-2 nucleic acid test carried out in our laboratory is a semi-quantitative test. Since SARS-CoV-2 nucleic acid was not detected in the early stages of the epidemic, it was not possible to evaluate the time and duration of the presence of nucleic acid in the stool, nor was it possible to evaluate the association between throat swab positivity and fecal positivity. Nevertheless, despite the low number of COVID-19 patients included in the present study and the small amount of available data on blood indices and the presence of viral nucleic acid, the preliminary characteristics of some blood indices are already obvious.

In summary, some Blood cell count indices upon hospital admission could be helpful to give some tips of diagnosis of SARS-CoV-2-infection、Flu A/B-infection and MP-infection; EO\# is associated with patient outcome and can be used as a reference for assessing the level of recovery. Meanwhile, EO\# is more strongly associated with the Ct value; in patients with COVID-19, fecal negativity is observed later than throat swab negativity, and when the throat swab turns negative, the patient's condition especially digestion is stable and The digestive tract is not sensitive to SARS-CoV-2, and SARS-CoV-2 in the intestinal tract has not been observed to be retransmitted to the respiratory tract. Due to inconsistencies in some blood indices and the fact it is unclear whether fecal-oral transmission takes place, there is a certain risk that patients are discharged before being fecal-negative.

Acknowledgments: We thank LetPub (www.letpub.com) for its linguistic assistance during the preparation of this manuscript.

Research funding: This study was supported by 2020 Research Project of Emergency Prevention and Control of 
New Coronavirus Infection and Pneumonia in Jinhua City (2020XG-02).

Author contributions: X.X.P. wrote the manuscript. C.S.Q. contributed to data analysis and created image attachments. C.W. collected the raw data from the laboratory information system. W.H.B. consulted on data interpretation and revised the manuscript. All authors contributed to the final version of the manuscript.

Competing interests: The authors report no conflict of interest. The authors alone are responsible for the content and the writing of the paper.

Informed consent: Paper informed consent has been obtained for each of the 17 patients using laboratory data. Ethical approval: The authors confirm that the ethical policies of the journal, as noted on the journal's author guidelines page, have been adhered to. The number of an ethics committee or institutional board approval is: 2020182-001.

Data availability statement: The data that support the findings of this study are available from JinHua Municipal Central Hospital. Restrictions apply to the availability of these data, which were used under license for this study. Data are available from the authors with the permission of JinHua Municipal Central Hospital.

\section{References}

1. Zhu N, Zhang D, Wang W, Li X, Yang B, Song J, et al. A novel coronavirus from patients with pneumonia in China, 2019. N Engl J Med 2020;382:727-33.

2. Zhou P, Yang XL, Wang XG, Hu B, Zhang L, Zhang W, et al. Discovery of a novel coronavirus associated with the recent pneumonia outbreak in humans and its potential bat origin. bioRxiv 2020 . https://doi.org/10.1101/2020.01.22.914952.

3. Rothe C, Schunk M, Sothmann P, Bretzel G, Froeschl G, Wallrauch $C$, et al. Transmission of SARS-COV-2 infection from an asymptomatic contact in Germany. N Engl J Med 2020;382:970-1.

4. Chu DKW, Pan Y, Cheng SMS, Hui KPY, Krishnan P, Liu Y, et al. Molecular diagnosis of a novel coronavirus (SARS-COV-2) causing an outbreak of pneumonia. Clin Chem 2020;66:549-55.

5. Huang C, Wang Y, Li X, Ren L, Zhao J, Hu Y, et al. Clinical features of patients infected with 2019 novel coronavirus in Wuhan, China. Lancet 2020;395:497-506.

6. Mo X, Qin W, Fu Q, Guan M. Understanding the influence factors in viral nucleic acid test of 2019 novel coronavirus (2019-nCoV) [J]. Chin J Lab Med 2020;43:E002.

7. World Health Organization. Laboratory diagnostics for novel coronavirus. WHO; 2020.

8. Cao B, Huang Y, She DY, Cheng QJ, Fan H, Tian XL, et al. Diagnosis and treatment of community-acquired pneumonia in adults: 2016 clinical practice guidelines by the Chinese Thoracic Society, Chinese Medical Association. Clin Respir J 2018;12:1320-60.
9. World Health Organization. Clinical management of severe acute respiratory infection (SARI) when novel coronavirus ( $\mathrm{nCoV}$ ) infection is suspected: interim guidance. WHO; 2020. License: CC BY-NC-SA 3.0 IGO.

10. Ai JW, Zhang HC, Xu T, Wu J, Zhu M, Yu YQ, et al. Optimizing diagnostic strategy for novel coronavirus pneumonia, a multicenter study in Eastern China. medRxiv 2020.

11. Zhou F, Yu T, Du R, Fan G, Liu Y, Liu Z, et al. Clinical course and risk factors for mortality of adult inpatients with COVID-19 in Wuhan, China: a retrospective cohort study. Lancet 2020;395:1054-62.

12. Zhang H, Kang Z, Gong H, Xu D, Wang G, Li Z, et al. The digestive system is a potential route of SARS-COV-2 infection: a bioinformatics analysis based on single-cell transcriptomes. BioRxiv 2020;69:1010-8.

13. Bertolini A, van de Peppel IP, Bodewes F, Moshage H, Fantin A, Farinati $F$, et al. Abnormal liver function tests in patients with COVID-19: relevance and potential pathogenesis. Hepatology 2020;72:1864-72.

14. Chan PK, Chen GG. Mechanisms of lymphocyte loss in SARS coronavirus infection. Hong Kong Med J 2008;14:21-6.

15. Altman LC, Hill JS, Hairfield WM, Mullarkey MF. Effects of corticosteroids on eosinophil chemotaxis and adherence. Clin Invest 1981;67:28-36.

16. Wardlaw AJ. Eosinophils in the 1990s: new perspectives on their role in health and disease. Postgrad Med 1994;70:536-52.

17. Percopo CM, Dyer KD, Karpe KA, Joseph BD, Helene FR. Eosinophils and respiratory virus infection: a dual-standard curve qRT-PCR-based method for determining virus recovery from mouse lung tissue. Methods Mol Biol 2014;1178:257-66.

18. Liu F, Xu A, Zhang Y, Xuan W, Yan T, Pan K, et al. Patients of COVID-19 may benefit from sustained lopinavir-combined regimen and the increase of eosinophil may predict the outcome of COVID-19 progression. Int J Infect Dis 2020;95:183-91.

19. Zhou J, Li C, Zhao G, Chu H, Wang D, Yan HH, et al. Human intestinal tract serves as an alternative infection route for Middle East respiratory syndrome coronavirus. Sci Adv 2017;3:eaao4966.

20. Cui J, Li F, Shi ZL. Origin and evolution of pathogenic coronaviruses. Nat Rev Microbiol 2019;17:181-92.

21. Markus H, Hannah KW, Nadine K, Müller M, Drosten C, Pöhlmann S. The novel coronavirus 2019 (SARS-COV-2) uses the SARS-coronavirus receptor ACE2 and the cellular protease TMPRSS2 for entry into target cells. bioRxiv 2020. https://doi. org/10.1101/2020.01.31.929042.

22. Michael L, Vincent $M$. Functional assessment of cell entry and receptor usage for lineage $B \beta$-coronaviruses, including SARS-COV-2. bioRxiv 2020. 2020.01.22.915660.

23. Holshue ML, DeBolt C, Lindquist S, Lofy KH, Wiesman J, Bruce H, et al. First case of 2019 novel coronavirus in the United States. N Engl J Med 2020;382:929-36.

24. World Health Organization. Laboratory testing for coronavirus disease 2019 (COVID-19) in suspected human cases: interim guidance, 2 March 2020. World Health Organization; 2020.

25. Mesoraca A, Margiotti K, Viola A, Cima A, Sparacino D, Giorlandino C. Evaluation of SARS-CoV-2 viral RNA in fecal samples. Virol J 2020;17:86.

26. Chen L, Lou J, Bai Y, Wang M. COVID-19 disease with positive fecal and negative pharyngeal and sputum viral tests. Am J Gastroenterol 2020;115:790. 